\title{
Metals Accumulation in Muscle Tissues and Digestive Contents of Periglypta reticulata (Kerang Geton) from Lancang Island, Jakarta
}

\author{
Suratno $^{1, *}$, Rachma Puspitasari $^{2}$, Zahriza Purnadayanti ${ }^{3}$, and Novita Sandra ${ }^{4}$ \\ ${ }^{1}$ Research Division for Natural Product Technology, Indonesian Institute of Sciences, Jl. Jogja-Wonosari Km 31.5, Gading, \\ Yogyakarta 55861, Indonesia \\ ${ }^{2}$ Research Center for Oceanography, Indonesian Institute of Sciences, Pasir Putih I, Ancol Timur, Jakarta 14430, Indonesia \\ ${ }^{3}$ Department of Fisheries and Marine Science, Brawijaya University, Jl. Veteran, Ketawanggede, Malang 65145, Indonesia \\ ${ }^{4}$ Toxicology Laboratory, Jakarta Public Health Laboratory, Jl. Rawasari Selatan No. 2, Cempaka Putih, Jakarta 10510, Indonesia
}

* Corresponding author:

tel: $+62-274-392570$

email: nanosan80@gmail.com

Received: September 2, 2019

Accepted: February 3, 2020

DOI: $10.22146 /$ ijc.49219

\begin{abstract}
The concentration of nineteen metals ( $\mathrm{Hg}, \mathrm{As}, \mathrm{Cd}, \mathrm{Co}, \mathrm{Cr}, \mathrm{Cu}, \mathrm{Fe}, \mathrm{Li}, \mathrm{Mg}, \mathrm{Mn}$, $\mathrm{Mo}, \mathrm{Ni}, \mathrm{Pb}, \mathrm{Se}, \mathrm{Sr}, \mathrm{Ti}, \mathrm{Tl}, \mathrm{V}$, and $\mathrm{Zn}$ ) were determined in muscle tissues and digestive contents of Periglypta reticulata (Kerang Geton), collected from Lancang Island part of Seribu Islands, Jakarta. An interaction between toxic and essential metal in a clam is also studied. The results showed high concentrations of As (4.56), $V(1.20)$, and $\mathrm{Zn}$ (4.91) $\mathrm{mg} / \mathrm{kg}$ wet weight in muscle tissues and As (7.16), Ti (2.53), and $\mathrm{Zn}$ (8.68) $\mathrm{mg} / \mathrm{kg}$ wet weight in digestive contents. Average concentrations of metals in muscle tissues and digestive contents were below regulation limit from permissible standard National Agency of Drug and Food Control except for Arsenic (As). The average concentration of metals in muscle tissues was significantly $(P<0.05)$ lower than in digestive contents except $\mathrm{As}, \mathrm{Co}, \mathrm{Cr}, \mathrm{Mg}$, and $\mathrm{Tl}$, respectively. Toxic metals $(\mathrm{Pb}, \mathrm{Hg}, \mathrm{Cd}$, and $\mathrm{As}$ ) showed a strong correlation with several essential metals so that these metals can be a threat to the main function of a particular metal. The present study showed digestive contents could accumulate in higher metals; therefore, we suggested removing it before consuming this clam.
\end{abstract}

Keywords: Periglypta reticulata; heavy metals; Lancang Island

\section{- INTRODUCTION}

Heavy metals contamination is one of the complex problems in an aquatic environment and raising concern over their potential in the risk of human health [1]. Some heavy metals (Co, $\mathrm{Cr}, \mathrm{Cu}, \mathrm{Fe}, \mathrm{Mn}, \mathrm{Mo}, \mathrm{Ni}, \mathrm{Se}, \mathrm{Zn}$ ) are essential elements for the organism being constituents of several key enzymes and playing important roles in various oxidation-reduction reactions [2]. However, an excess amount of metals may produce cellular and tissue damage [3]. Metals such as $\mathrm{Hg}, \mathrm{Pb}, \mathrm{Cd}$, and As have no established biological functions and are considered as non-essential and potentially toxic at relatively low concentrations [4]. The heavy metals concentration may be attributed to sewage and agricultural drainage. Besides, the corrosion of the ship's hull coating and antifouling paints at the ship can cause a high concentration of copper in the lake [5].

Marine bivalves could accumulate metals presented at undetectable levels from environmental to very high concentrations in tissues [6]. Bivalves are known to feed on suspended particles in the water column, which could be contaminated by various contaminants derived from either anthropogenic activities or natural emissions [7]. The primary source of heavy metals contamination in the natural environment is the run-off from agricultural activities [8]. Bivalves are known to filter between twenty and one hundred litters of surrounding waters a day. In doing so, they accumulate natural or anthropogenic contaminants [9].

Furthermore, since the bivalves have high nutritional and economic values, eating bivalves contain 
high concentrations of heavy metals is dangerous and threats to human health [10]. The present study was aimed to investigate the concentration of toxic metals (As, $\mathrm{Pb}, \mathrm{Cd}$, and $\mathrm{Hg}$ ) and essential metals $(\mathrm{Co}, \mathrm{Cr}, \mathrm{Cu}, \mathrm{Fe}, \mathrm{Li}$, $\mathrm{Mg}, \mathrm{Mn}, \mathrm{Mo}, \mathrm{Ni}, \mathrm{Se}, \mathrm{Sr}, \mathrm{Ti}, \mathrm{Tl}, \mathrm{V}$, and $\mathrm{Zn}$ ) in muscle tissues and digestive content of Periglypta reticulata from Lancang Island, part of Seribu Islands, Jakarta. The other goal was to study the interaction between toxic and essential metals in the clam. Lancang Island located $30 \mathrm{~km}$ North-West from Jakarta Bay and $9 \mathrm{~km}$ from Cisadane estuary in Tangerang. Jakarta Bay receives enormous amounts of municipal discharges from the Jakarta metropolis [11] that caused marine pollution with various pollutants, especially heavy metals.

\section{- EXPERIMENTAL SECTION}

\section{Materials}

Periglypta reticulata were purchased from fisherman in Lancang Island and directly placed in a ziplock plastic bag and freeze until further analysis. All reagents used were analytical-reagents grade. The solutions were prepared using ultra-pure water (Milli-Q), Nitric acid (65\% $\mathrm{HNO}_{3}$ Suprapur, Merck), $\mathrm{HCl} 37 \%$ (Merck), Hg standard solution $1.000 \mathrm{mg} / \mathrm{L}$ (Merck) and L-Cysteine (Nacalai Tesque Inc. Japan).

\section{Instrumentation}

Caliper and analytical balance (Sartorius BP 210 S) were used for morphometric analysis. Dried samples were prepared using oven (Heraeus Instrument), petri dish, spatula, mortar, and pestle. ICP-OES 7400 Thermo and Mercury Analyzer NIC MA-3000 were used for concentrations analysis.

\section{Procedure}

\section{Sample collection}

In the laboratory, bivalves were cleaned to remove dirt attached to the bivalve and were separated from muscle tissues and digestive contents. Morphometric analysis of the total weight of the wet tissues was measured with Sartorius analytical balance, while the length and width of bivalves were measured with a digital caliper. Samples were dried in an oven at $60^{\circ} \mathrm{C}$ for $24 \mathrm{~h}$ for heavy metals analysis. Moisture contents of muscle tissues and digestive contents were measured with oven-dried at $105^{\circ} \mathrm{C}$ for $24 \mathrm{~h} \mathrm{[12].} \mathrm{Muscle} \mathrm{tissues} \mathrm{(MT)} \mathrm{and} \mathrm{digestive}$ contents (DC) were ground into a fine powder with mortar and pestle for the subsequent analysis.

\section{Metal analysis}

Three replicates of dried MT and DC samples were individually weighed approximately $10-20 \mathrm{mg}$ directly in the sample boat and analyzed with mercury analyzer for total mercury concentration. The modified method from USEPA 3051a for total metals analysis was utilizing a mixture of concentrated $\mathrm{HNO}_{3}$ and $\mathrm{HCl}$ and a microwave oven [13]. In brief, the mixture of acid $(9 \mathrm{~mL}$ $\mathrm{HNO}_{3}$ and $3 \mathrm{~mL} \mathrm{HCl}$ ) was drop-added into $0.5 \mathrm{~g}$ of samples then the mixture was heated using microwave oven CEM MARS 5 Express at $185{ }^{\circ} \mathrm{C}$ for $15 \mathrm{~min}$ and hold for $30 \mathrm{~min}$. Then the solution was filtered using Whatman filter paper No. 41, and the filtrate was diluted to $25 \mathrm{~mL}$ using deionized water. Three replicates of dried MT and DC samples were also applied for a non- $\mathrm{Hg}$ measurement. The samples were measured using ICPOES 7400 Thermo for metals analysis.

\section{Data analysis}

Statistical correlation analysis was based on IBM SPSS Statistics 22. Pearson's correlation coefficient analysis was used to determine the significant relationship between metal accumulation in muscle tissues and digestive contents with two-tailed $p$-value $<0.05$ and $p$-value $<0.01$ were considered significant. All graphical plot was based on the R program.

\section{- RESULTS AND DISCUSSION}

Details of metals concentration MT and DC in $P$. reticulata are shown in Fig. 1 and Fig. 2. Bivalves ( $\mathrm{n}=10$ individual samples) collected in this study displayed similar-sized of $8.00 \pm 0.53 \mathrm{~cm}$ (Length) $\times 8.65 \pm 0.85 \mathrm{~cm}$ (Width). The average wet weights of total tissues were $32.40 \pm 8.83 \mathrm{~g}$, and the moisture content for MT and DC were $81.38 \%$ and $75.64 \%$, respectively. Concentrations of As, Co, Cr, $\mathrm{Mg}$, and $\mathrm{Tl}$ were not statistically significantly different between MT and DC. Concentrations of toxic metals $(\mathrm{Hg}, \mathrm{Pb}$, and $\mathrm{Cd}$ ) except As were lower than the permissible limit from Badan Pengawas Obat dan Makanan or National Agency of 

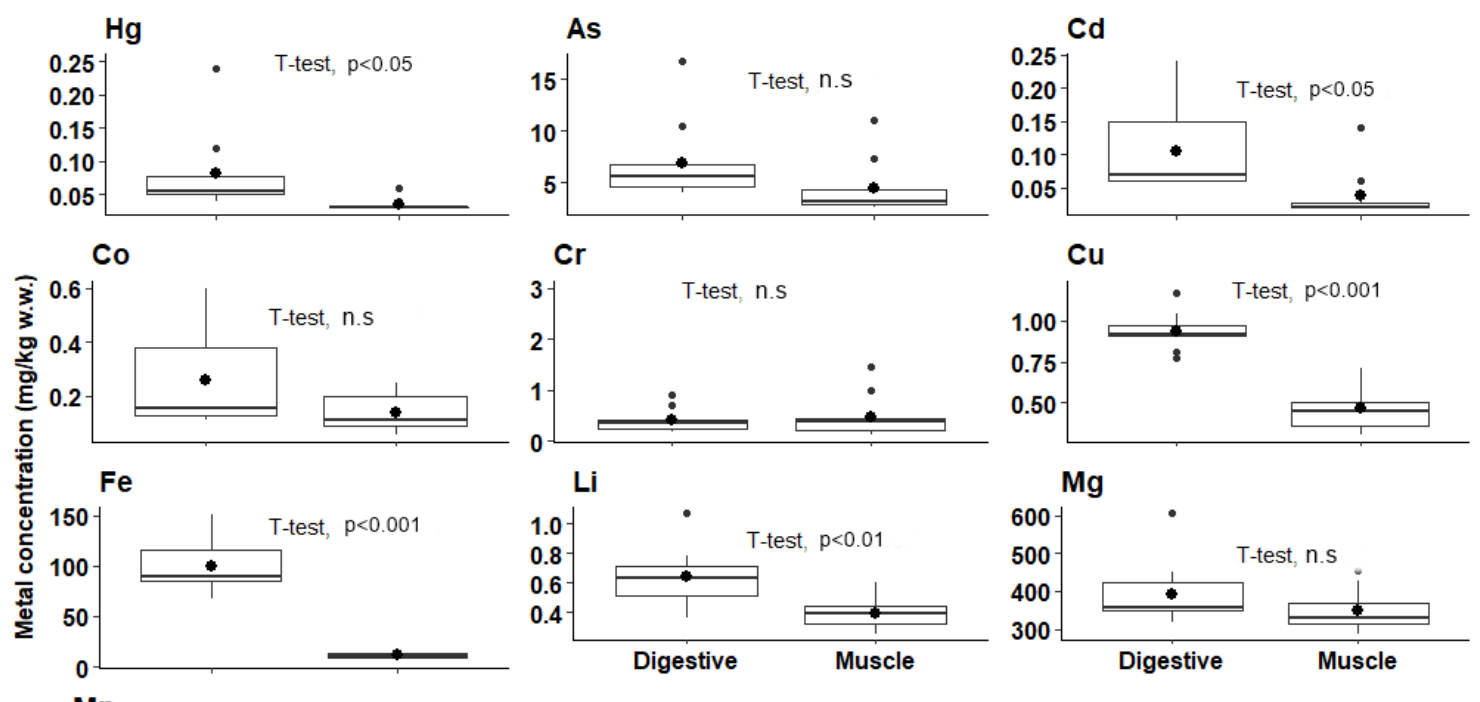

$$
\mathrm{Mn}
$$

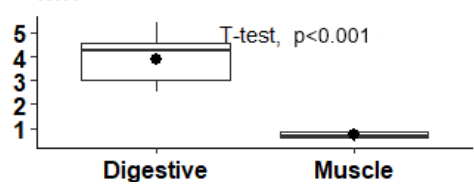

Fig 1. Metals concentrations (mg/kg w.w.) in digestive contents and muscle tissues of $P$. reticulata (“•” represents mean value)
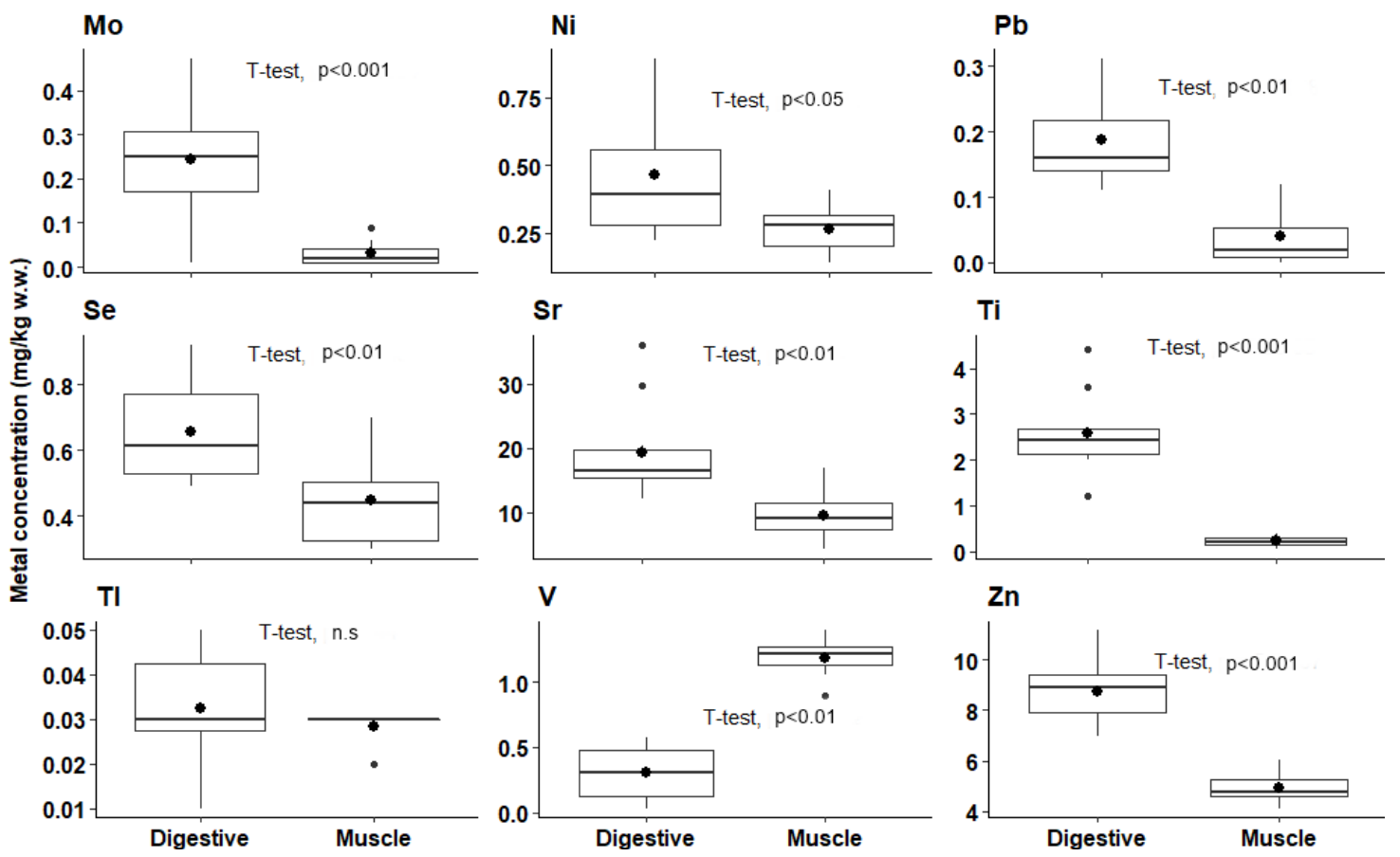

Fig 2. Metals concentrations ( $\mathrm{mg} / \mathrm{kg}$ w.w.) in digestive contents and muscle tissues of $P$. reticulata(“•” represents mean value)

Drug and Food Control (NADFC) in muscle tissues and digestive contents. Concentrations of $\mathrm{As}, \mathrm{Co}, \mathrm{Cr}, \mathrm{Mg}$ and

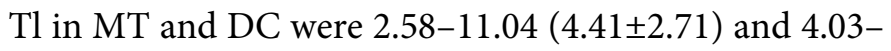

$16.74(6.93 \pm 3.93) ; 0.06-0.25(0.14 \pm 0.07)$ and $0.11-0.60$

$(0.26 \pm 0.18) ; \quad 0.14-1.45 \quad(0.49 \pm 0.42)$ and $0.18-0.90$ $(0.41 \pm 0.23) ; 288.86-454.28(349.70 \pm 53.58)$ and $318.90-$ 
605.27 (393.56 \pm 85.98$) ; 0.06-0.37(0.22 \pm 0.11)$ and $0.014-$ $0.053(0.033 \pm 0.014) \mathrm{mg} / \mathrm{kg}$ w.w., respectively. In this study, the concentration of Arsenic (As) in MT and DC (2.58$11.04(4.41 \pm 2.71)$ and $4.03-16.74(6.93 \pm 3.93) \mathrm{mg} / \mathrm{kg}$ w.w. were higher compared to study that reported by Copat et al. [14] in Donax trunculus from Catalina Gulf, Italia $(1.53 \mathrm{mg} / \mathrm{kg}$ w.w.), Sivaperumal et al. [15] in Villorita cyprinoides from Cochin area, India $(0.69 \mathrm{mg} / \mathrm{kg}$ w.w.), Lei at el. [16] in Mactra chinensis from Shanghai $(0.20 \mathrm{mg} / \mathrm{kg}$ d.w. $)$ and Li et al. [17] in Mactra veneriformis from Bohai Bay, China (1.44-2.51 mg/kg w.w.). These facts showed an As enrichment in $P$. reticulata that was not fully understood because of sediment, as the main factor, was not examined in this study. Enrichment As could be attributed to the natural and anthropogenic sources. Natural sources mostly related to phosphate deposits and anthropogenic As can be driven As-rich pesticides in the past and phosphorite processing activities [17].

Concentrations of $\mathrm{Hg}, \mathrm{Cd}$, and $\mathrm{Ni}$ were statistically significant $(p<0.05)$; Li, $\mathrm{Pb}, \mathrm{Se}, \mathrm{Sr}$, and V statistically significant $(p<0.01)$ different between MT and DC.
Concentrations of $\mathrm{Cu}, \mathrm{Fe}, \mathrm{Mn}, \mathrm{Mo}, \mathrm{Ti}$, and $\mathrm{Zn}$ were statistically highly significant $(p<0.001)$ different between MT and DC in $P$. reticulata. Copper, iron, manganese, molybdenum, and zinc were known as cofactors of many enzymes that play an important role in aquatic organisms [17]. Iron is an essential element for biological function, but in excess, it is related to heart disease, cancer, and impaired insulin sensitivity [18]. $\mathrm{Mn}$ is an essential micronutrient for many biochemical reactions in living beings [19]. Zinc has generally been considered to be non-toxic and a minor nutrient required for growth and development [20].

Accumulation of heavy metals in marine mollusks can be affected by many factors, including endogenous and exogenous factors. Endogenous factors such as body size, growth, fitness, reproductive condition and genotypes [21], the differences in biokinetic uptake, depuration rate, and other physiological processes could contribute to the variations in the heavy metal concentration in tissues [22]. Meanwhile, the exogenous factors could be salinity, metal bioavailability [23], alkalinity, and others [19].

Table 1. Pearson correlation R between metals concentration ( $\mathrm{mg} / \mathrm{kg} \mathrm{w} . \mathrm{w}$.$) in muscle tissues of P$. reticulata

\begin{tabular}{|c|c|c|c|c|c|c|c|c|c|c|c|c|}
\hline Element & $\mathrm{Hg}$ & As & $\mathrm{Cd}$ & Co & $\mathrm{Cr}$ & $\mathrm{Cu}$ & $\mathrm{Fe}$ & $\mathrm{Li}$ & $\mathrm{Mg}$ & $\mathrm{Mn}$ & Mo & $\mathrm{Ni}$ \\
\hline $\mathrm{Hg}$ & & $0.783^{a}$ & & 0.431 & & -0.252 & & 0.480 & -0.351 & 0.440 & -0.202 & $0.652^{b}$ \\
\hline As & & & & $0.648^{b}$ & & & & & -0.466 & 0.255 & 0.323 & 0.603 \\
\hline $\mathrm{Cd}$ & & & & & 0.425 & 0.440 & 0.585 & & -0.294 & -0.446 & 0.475 & -0.231 \\
\hline Co & & & & & & -0.441 & & 0.370 & & 0.358 & 0.350 & $0.761^{b}$ \\
\hline $\mathrm{Cr}$ & & & & & & $0.769^{a}$ & $0.684^{b}$ & & -0.257 & & 0.234 & \\
\hline $\mathrm{Cu}$ & & & & & & & & -0.286 & -0.509 & -0.299 & 0.409 & -0.479 \\
\hline $\mathrm{Fe}$ & & & & & & & & 0.448 & & & & 0.263 \\
\hline $\mathrm{Li}$ & & & & & & & & & 0.495 & 0.382 & -0.472 & 0.575 \\
\hline $\mathrm{Mg}$ & & & & & & & & & & & -0.314 & \\
\hline $\mathrm{Mn}$ & & & & & & & & & & & -0.466 & $0.716^{b}$ \\
\hline \multicolumn{13}{|l|}{ Mo } \\
\hline \multicolumn{13}{|l|}{$\mathrm{Ni}$} \\
\hline \multicolumn{13}{|l|}{$\mathrm{Pb}$} \\
\hline \multicolumn{13}{|l|}{$\mathrm{Se}$} \\
\hline \multicolumn{13}{|l|}{$\mathrm{Sr}$} \\
\hline \multicolumn{13}{|l|}{$\mathrm{Ti}$} \\
\hline \multicolumn{13}{|l|}{$\mathrm{Tl}$} \\
\hline \multicolumn{13}{|l|}{$\mathrm{V}$} \\
\hline $\mathrm{Zn}$ & & & & & & & & & & & & \\
\hline
\end{tabular}


Table 1. Pearson correlation R between metals concentration ( $\mathrm{mg} / \mathrm{kg}$ w.w.) in muscle tissues of P. reticulata (Continued)

\begin{tabular}{|c|c|c|c|c|c|c|c|}
\hline Element & $\mathrm{Pb}$ & $\mathrm{Se}$ & $\mathrm{Sr}$ & $\mathrm{Ti}$ & $\mathrm{Tl}$ & $\mathrm{V}$ & $\mathrm{Zn}$ \\
\hline $\mathrm{Hg}$ & & & -0.297 & & 0.279 & & -0.537 \\
\hline As & -0.260 & & & & & & \\
\hline $\mathrm{Cd}$ & -0.201 & $0.674^{b}$ & & & -0.353 & & \\
\hline Co & & & & & & 0.430 & \\
\hline $\mathrm{Cr}$ & -0.224 & 0.427 & & 0.329 & -0.627 & & -0.212 \\
\hline $\mathrm{Cu}$ & -0.423 & 0.512 & & 0.416 & $-0.737^{b}$ & & \\
\hline $\mathrm{Fe}$ & & 0.246 & & & -0.305 & & \\
\hline $\mathrm{Li}$ & & -0.511 & -0.512 & & 0.406 & & \\
\hline $\mathrm{Mg}$ & 0.355 & -0.565 & & -0.334 & 0.398 & & 0.424 \\
\hline Mn & & & & 0.201 & & -0.204 & -0.439 \\
\hline Mo & -0.370 & 0.477 & 0.354 & & $0.719^{b}$ & 0.625 & 0.449 \\
\hline $\mathrm{Ni}$ & 0.225 & -0.337 & & & & -0.206 & -0.237 \\
\hline $\mathrm{Pb}$ & & -0.480 & & & 0.416 & $-0.716^{b}$ & \\
\hline Se & & & 0.500 & & -0.603 & 0.400 & -0.344 \\
\hline Sr & & & & 0.216 & -0.536 & 0.287 & \\
\hline $\mathrm{Ti}$ & & & & & -0.417 & -0.334 & \\
\hline $\mathrm{Tl}$ & & & & & & -0.404 & \\
\hline V & & & & & & & 0.417 \\
\hline $\mathrm{Zn}$ & & & & & & & \\
\hline
\end{tabular}

Only meaningful $|\mathrm{R}|>0.2$ correlation is shown; Bold is significant correlation value of $>0.4$

${ }^{\mathrm{a}}$ Correlation is significant at the 0.01 level (2-tailed); ${ }^{\mathrm{b}}$ Correlation is significant at the 0.05 level (2-tailed)
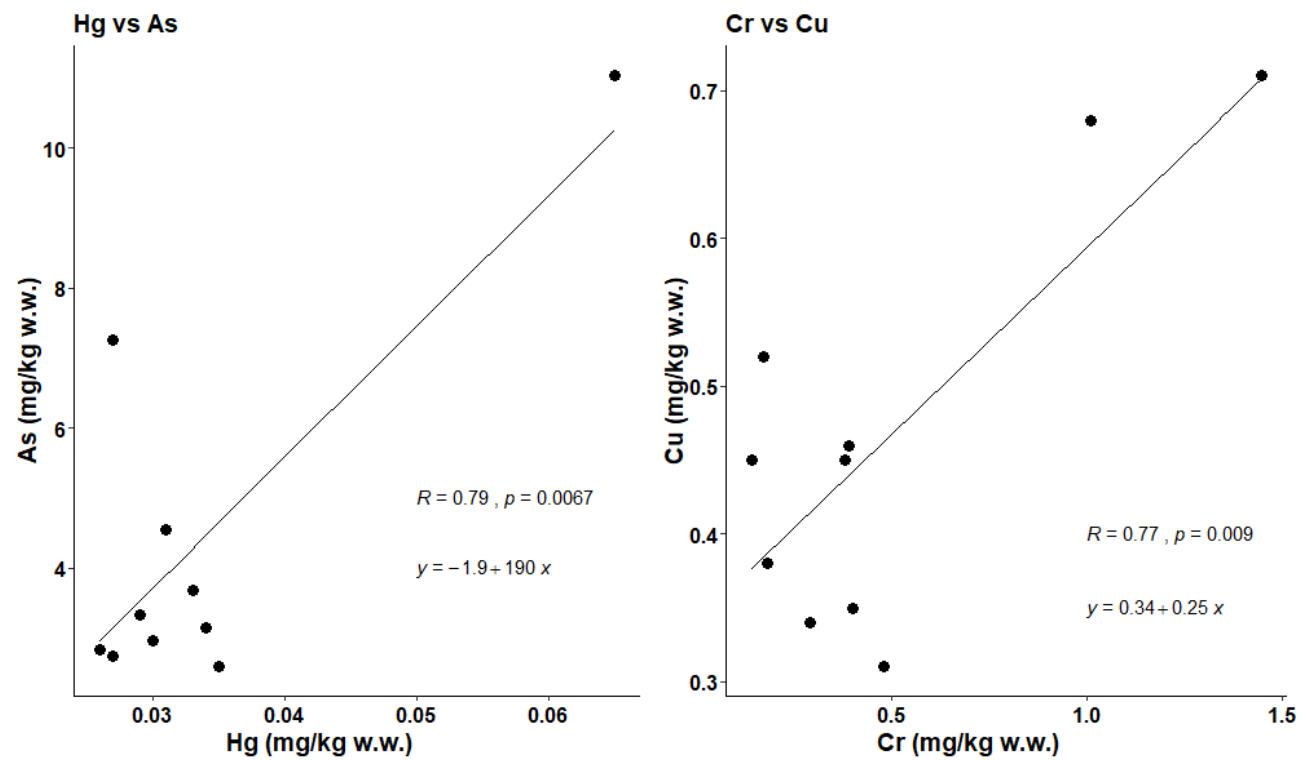

Fig 3. Elemental relationship in the muscle tissues of $P$. reticulata (wet weight)

Pearson correlation between metals in muscle tissues in P. reticulata showed in Table 1 and a significantly positive linear correlation $(p<0.05)$ were observed between concentrations of some metals (As-Co, Cr-Fe,
Hg-Ni, Co-Ni, Mn-Ni, Cd-Se, Mo-Tl) and significantly negative linear correlation $(p<0.05)(\mathrm{Cu}-\mathrm{Tl}$ and $\mathrm{Pb}-\mathrm{V})$. A significant positive correlation $(p<0.01)$ between $\mathrm{Hg}$ As $\left(r^{2}=0.79\right)$ and $\mathrm{Cr}-\mathrm{Cu}\left(r^{2}=0.77\right)$ is shown in Fig. 3 . 
Table 2. Pearson correlation R between metals concentration ( $\mathrm{mg} / \mathrm{kg}$ w.w.) in digestive contents of $P$. reticulata

\begin{tabular}{|c|c|c|c|c|c|c|c|c|c|c|c|}
\hline Element & $\mathrm{Hg}$ & As & $\mathrm{Cd}$ & $\mathrm{Co}$ & $\mathrm{Cr}$ & $\mathrm{Cu}$ & $\mathrm{Fe}$ & $\mathrm{Li}$ & $\mathrm{Mg}$ & $\mathrm{Mn}$ & Mo \\
\hline \multicolumn{12}{|l|}{$\mathrm{Hg}$} \\
\hline As & $0.976^{\mathrm{a}}$ & & & & & & & & & & \\
\hline $\mathrm{Cd}$ & $0.895^{a}$ & $0.895^{\mathrm{a}}$ & & & & & & & & & \\
\hline Co & $0.676^{b}$ & $0.684^{b}$ & $0.923^{\mathrm{a}}$ & & & & & & & & \\
\hline $\mathrm{Cr}$ & & & 0.226 & 0.350 & & & & & & & \\
\hline \multirow[t]{2}{*}{$\mathrm{Cu}$} & - & -0.529 & -0.255 & & & & & & & & \\
\hline & 0.411 & & & & & & & & & & \\
\hline $\mathrm{Fe}$ & & & 0.298 & 0.431 & 0.622 & 0.601 & & & & & \\
\hline $\mathrm{Li}$ & 0.202 & & 0.411 & 0.534 & 0.319 & 0.459 & $0.813^{a}$ & & & & \\
\hline $\mathrm{Mg}$ & & -0.220 & 0.209 & 0.487 & & 0.631 & $0.686^{b}$ & $0.701^{b}$ & & & \\
\hline \multirow[t]{2}{*}{$\mathrm{Mn}$} & - & -0.574 & -0.374 & & 0.373 & 0.477 & 0.605 & 0.547 & 0.388 & & \\
\hline & 0.575 & & & & & & & & & & \\
\hline Mo & 0.477 & 0.478 & $0.724^{b}$ & $0.770^{a}$ & & & 0.480 & 0.509 & 0.443 & & \\
\hline $\mathrm{Ni}$ & $0.716^{b}$ & $0.701^{b}$ & $0.910^{\mathrm{a}}$ & $0.969^{a}$ & 0.272 & & 0.395 & 0.558 & 0.500 & -0.222 & $0.753^{b}$ \\
\hline $\mathrm{Pb}$ & 0.411 & 0.420 & $0.707^{b}$ & $0.793^{a}$ & 0.546 & 0.223 & $0.654^{b}$ & 0.500 & 0.475 & & 0.606 \\
\hline $\mathrm{Se}$ & 0.534 & 0.577 & 0.594 & 0.618 & & -0.556 & & 0.255 & & & $0.660^{b}$ \\
\hline $\mathrm{Sr}$ & $0.709^{b}$ & $0.656^{b}$ & $0.741^{b}$ & 0.735 & 0.360 & -0.236 & 0.424 & 0.628 & 0.337 & & 0.426 \\
\hline $\mathrm{Ti}$ & -0.207 & -0.294 & & -0.257 & & $0.733^{b}$ & 0.560 & 0.226 & & 0.404 & \\
\hline $\mathrm{Tl}$ & -0.293 & -0.408 & & 0.218 & & 0.499 & 0.355 & & $0.775^{b}$ & & \\
\hline V & 0.467 & 0.554 & 0.525 & 0.578 & 0.614 & $-0.998^{a}$ & & -0.288 & -0.824 & -0.331 & 0.910 \\
\hline $\mathrm{Zn}$ & -0.285 & -0.283 & & 0.286 & 0.277 & $0.675^{b}$ & $0.705^{b}$ & $0.683^{b}$ & $0.653^{b}$ & $0.681^{b}$ & 0.387 \\
\hline
\end{tabular}

Table 2. Pearson correlation R between metals concentration ( $\mathrm{mg} / \mathrm{kg}$ w.w.) in digestive contents of P. reticulata (Continued)

\begin{tabular}{|c|c|c|c|c|c|c|c|c|}
\hline Element & $\mathrm{Ni}$ & $\mathrm{Pb}$ & $\mathrm{Se}$ & $\mathrm{Sr}$ & $\mathrm{Ti}$ & $\mathrm{Tl}$ & $\mathrm{V}$ & $\mathrm{Zn}$ \\
\hline \multicolumn{9}{|l|}{$\mathrm{Hg}$} \\
\hline \multicolumn{9}{|l|}{ As } \\
\hline \multicolumn{9}{|l|}{$\mathrm{Cd}$} \\
\hline \multicolumn{9}{|l|}{ Co } \\
\hline \multicolumn{9}{|l|}{$\mathrm{Cr}$} \\
\hline \multicolumn{9}{|l|}{$\mathrm{Cu}$} \\
\hline \multicolumn{9}{|l|}{$\mathrm{Fe}$} \\
\hline \multicolumn{9}{|l|}{$\mathrm{Li}$} \\
\hline \multicolumn{9}{|l|}{ Mg } \\
\hline \multicolumn{9}{|l|}{$\mathrm{Mn}$} \\
\hline \multirow{2}{*}{\multicolumn{9}{|c|}{$\begin{array}{l}\text { Mo } \\
\mathrm{Ni}\end{array}$}} \\
\hline & & & & & & & & \\
\hline $\mathrm{Pb}$ & $0.663^{b}$ & & & & & & & \\
\hline $\mathrm{Se}$ & $0.690^{b}$ & & & & & & & \\
\hline $\mathrm{Sr}$ & $0.806^{\mathrm{a}}$ & 0.395 & 0.606 & & & & & \\
\hline $\mathrm{Ti}$ & -0.330 & 0.270 & -0.575 & -0.334 & & & & \\
\hline $\mathrm{Tl}$ & & 0.338 & -0.236 & & & & & \\
\hline V & 0.567 & & $0.987^{b}$ & 0.251 & -0.660 & -0.891 & & \\
\hline $\mathrm{Zn}$ & & 0.604 & & & 0.507 & 0.330 & -0.624 & \\
\hline
\end{tabular}

Only meaningful $|\mathrm{R}|>0.2$ correlation is shown; Bold is significant correlation value of $>0.4$

a Correlation is significant at the 0.01 level (2-tailed); ${ }^{\mathrm{b}}$ Correlation is significant at the 0.05 level (2-tailed)

Pearson correlation between metals in digestive contents in $P$. reticulata showed in Table 2 and a significantly positive linear correlation $(p<0.05)$ were observed between concentrations of some metals ( $\mathrm{Hg}-\mathrm{Co}$, 

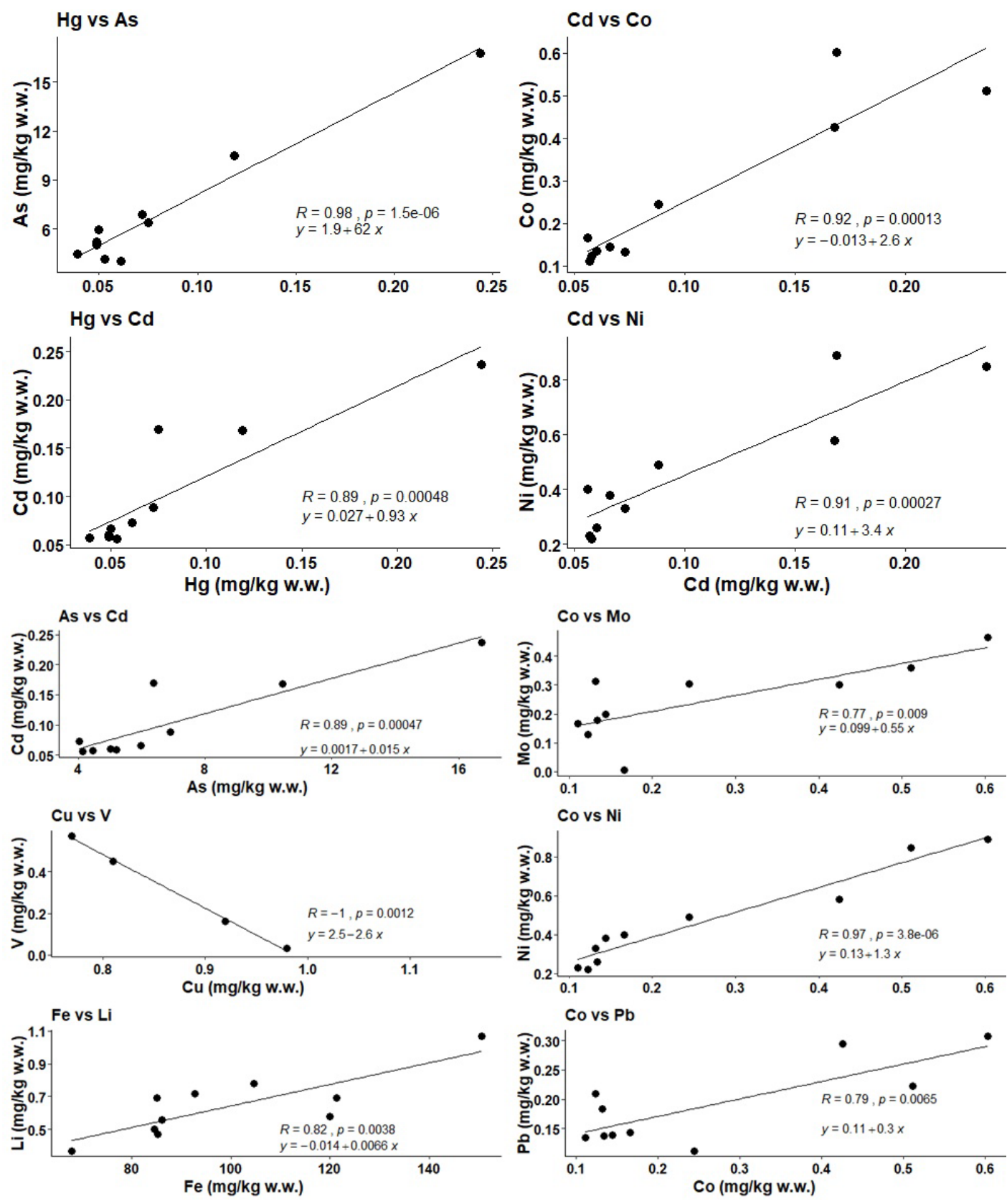

Fig 4. Elemental relationship in the digestive contents of $P$. reticulata (wet weight)

Hg-Ni, Hg-Sr, As-Co, As-Ni, As-Sr, Cd-Mo, Cd-Pb, Cd$\mathrm{Sr}, \mathrm{Cu}-\mathrm{Ti}, \mathrm{Cu}-\mathrm{Zn}$, Fe-Mg, Fe-Pb, Fe-Zn, Li-Mg, Li-Zn, Mg-Tl, Mg-Zn, Mn-Zn, Mo-Ni, Mo-Se, Ni-Pb, Ni-Se, and $\mathrm{Se}-\mathrm{V})$. A significantly positive linear correlation $(p<0.01)$ between Hg-As $\left(r^{2}=0.98\right), \mathrm{Hg}-\mathrm{Cd}\left(\mathrm{r}^{2}=0.89\right), \mathrm{Cd}-\mathrm{Co}\left(\mathrm{r}^{2}=\right.$ $0.92)$, Cd-Ni $\left(r^{2}=0.91\right)$, As-Cd $\left(r^{2}=0.89\right)$, Co-Mo $\left(r^{2}=\right.$ $0.77)$, Co-Ni $\left(r^{2}=0.97\right)$, Fe-Li $\left(r^{2}=0.82\right) \mathrm{Co}-\mathrm{Pb}\left(\mathrm{r}^{2}=0.79\right)$ and significantly negative correlation $(p<0.01)$ for $\mathrm{Cu}-\mathrm{V}$ $\left(r^{2}=-1\right)$ are shown in Fig. 4. Copper and Titanium showed a positive correlation in the digestive contents of $P$. reticulata. Therefore, it indicated that these metals sources come from slow corrosion of anti-fouling coating paint in a boat [24]. Concentrations of heavy metals $P$. reticulata from Lancang Island compared to the previously reported from around the world and guidelines (NADFC, ANZFC, and JECFA/WHO) were shown in Table. 3.

In the digestive content, $\mathrm{Cd}$ as a toxic element was positively correlated with the most of essential metals, Mo, 
Table 3. Comparison of heavy metal concentrations in muscle tissue and digestive contents of $P$. reticulata from Lancang Island and Veniridae clams in other area (metal concentration in $\mathrm{mg} / \mathrm{kg}$; dry weight (d.w.) or wet weight (w.w.) basis; bold indicated exceeding maximum limit from NADFC 2018)

\begin{tabular}{|c|c|c|c|c|c|c|c|c|c|c|c|c|c|c|}
\hline Site & Species & & Year & $\mathrm{Hg}$ & As & $\mathrm{Cd}$ & Co & $\mathrm{Cr}$ & $\mathrm{Cu}$ & $\mathrm{Fe}$ & $\mathrm{Li}$ & $\mathrm{Mg}$ & $\mathrm{Mn}$ & Mo \\
\hline \multirow[t]{2}{*}{$\begin{array}{l}\text { Lancang Island, } \\
\text { Indonesia }\end{array}$} & $\begin{array}{l}\text { Periglypta reticulata } \\
\text { (muscle) }\end{array}$ & w.w. & 2018 & 0.03 & 4.41 & 0.04 & 0.14 & 0.49 & 0.47 & 11.59 & 0.39 & 349.70 & 0.71 & 0.03 \\
\hline & $\begin{array}{l}\text { P. reticulata } \\
\text { (digestive) }\end{array}$ & w.w. & 2018 & 0.08 & 6.93 & 0.10 & 0.26 & 0.41 & 0.94 & 99.87 & 0.64 & 393.56 & 3.91 & 0.24 \\
\hline Catania Gulf & $\begin{array}{l}\text { D. trunculus } \\
\text { (composite) }\end{array}$ & w.w. & 2012 & & 1.53 & 0.01 & & 0.25 & & & & & 4.26 & \\
\hline Cochin Area & $\begin{array}{l}\text { Villorita crypinoides } \\
\text { (composite) }\end{array}$ & w.w. & 2011 & & & 0.34 & & 0.26 & 1.4 & & & & & \\
\hline Shanghai & $\begin{array}{l}\text { Mactra chinensis } \\
\text { (composite) }\end{array}$ & d.w. & 2008 & & 0.20 & 0.03 & & 0.10 & & & & & 5.18 & \\
\hline Bohai Bay & $\begin{array}{l}\text { Mactra veneriformis } \\
\text { (soft tissue) }\end{array}$ & w.w. & 2008 & & 1.44 & 0.27 & 0.55 & 1.37 & 2.34 & & & & 23.95 & 0.12 \\
\hline Parit Jawa, Johor & $\begin{array}{l}\text { Polymesoda erosa } \\
\text { (total tissue) }\end{array}$ & d.w. & 2009 & & & 3.26 & & & 14.1 & 790 & & & & \\
\hline Parit Jawa, Johor & $\begin{array}{l}\text { Polymesoda erosa } \\
\text { (foot) }\end{array}$ & d.w. & 2009 & & & 1.47 & & & 3.77 & 110 & & & & \\
\hline Parit Jawa, Johor & $\begin{array}{l}\text { Polymesoda erosa } \\
\text { (gill) }\end{array}$ & d.w. & & & & & 1.46 & & 12.8 & 1088 & & & & \\
\hline Telok Mas, Mallaca & $\begin{array}{l}\text { Polymesoda erosa } \\
\text { (total tissue) }\end{array}$ & d.w. & 2009 & & & 4.23 & 2.96 & & 15.7 & 1111 & & & & \\
\hline Telok Mas, Mallaca & $\begin{array}{l}\text { Polymesoda erosa } \\
\text { (foot) }\end{array}$ & d.w. & 2009 & & & & 0.253 & & 1.80 & & 93.5 & & & \\
\hline Telok Mas, Mallaca & $\begin{array}{l}\text { Polymesoda erosa } \\
\text { (gill) }\end{array}$ & d.w. & 2009 & & & & 2.86 & & 8.81 & 1620 & & & & \\
\hline $\begin{array}{l}\text { Coast of New } \\
\text { Celedonia }\end{array}$ & $\begin{array}{l}\text { Gafrarium tumidum } \\
\text { (soft tissue) }\end{array}$ & w.w. & 2008 & 0.42 & & & & & & & & & & \\
\hline $\begin{array}{l}\text { Coast of New } \\
\text { Celedonia }\end{array}$ & $\begin{array}{l}\text { Periglypta chemnitzi } \\
\text { (soft tissue) }\end{array}$ & w.w. & 2008 & 0.4 & & & & & & & & & & \\
\hline Astamudi Lagoon & $\begin{array}{l}\text { Villorita cyprinoides } \\
\text { (soft tissue) }\end{array}$ & d.w. & 2017 & & & 0.08 & 0.87 & 3.57 & 17.73 & 69.23 & & 574.99 & 5.16 & \\
\hline $\begin{array}{l}\text { Catania Fish } \\
\text { Market }\end{array}$ & $\begin{array}{l}\text { D. trunculus } \\
\text { (composite) }\end{array}$ & w.w. & 2013 & & 1.52 & 0.005 & & 0.24 & & & & & 4.25 & \\
\hline Marudu Bay & $\begin{array}{l}\text { Polymesoda expansa } \\
\text { (soft tissue) }\end{array}$ & d.w. & 2017 & & 9.12 & & & 6.07 & 16.6 & & & & 138.1 & \\
\hline $\begin{array}{l}\text { Rias of Pontevedra } \\
\text { (A1) }\end{array}$ & $\begin{array}{l}\text { Dosiona exoleta } \\
\text { (soft tissue) }\end{array}$ & d.w. & 2006 & & & 0.29 & & & 8.67 & & & & & \\
\hline (A2) & & d.w. & & & & 0.25 & & & 9.13 & & & & & \\
\hline (A4) & & d.w. & & & & 0.75 & & & 16.10 & & & & & \\
\hline $\begin{array}{l}\text { Kuala Kemaman, } \\
\text { Terengganu, }\end{array}$ & Polymesoda expansa & d.w. & 2016 & & & & & & & & & & & \\
\hline Coastline of India & Polymesoda aerosa & d.w. & 2013 & & & & & & & & & & & \\
\hline \multirow[t]{4}{*}{$\begin{array}{l}\text { Tanjung Lumpur, } \\
\text { Malaysia }\end{array}$} & $\begin{array}{l}\text { Polymesoda expansa } \\
\text { (soft tissue) }\end{array}$ & d.w. & 2017 & & & & & & & & & & & \\
\hline & NADFC (2018) & w.w. & 2018 & 0.50 & 0.25 & 0.10 & & & & & & & & \\
\hline & ANZFC & w.w. & 2000 & 0.5 & 1 & 2 & & & & & & & & \\
\hline & JECFA/WHO & w.w. & 2016 & 0.5 & & 2 & & & & & & & & \\
\hline
\end{tabular}


Table 3. Comparison of heavy metal concentrations in muscle tissue and digestive contents of $P$. reticulata from Lancang Island and Veniridae clams in other area (metal concentration in $\mathrm{mg} / \mathrm{kg}$; dry weight (d.w.) or wet weight (w.w.) basis; bold indicated exceeding maximum limit from NADFC 2018) (Continued)

\begin{tabular}{|c|c|c|c|c|c|c|c|c|c|c|c|c|c|}
\hline Site & Species & & Year & $\mathrm{Ni}$ & $\mathrm{Pb}$ & $\mathrm{Sb}$ & $\mathrm{Se}$ & $\mathrm{Sr}$ & $\mathrm{Ti}$ & $\mathrm{Tl}$ & $\mathrm{V}$ & $\mathrm{Zn}$ & Ref. \\
\hline \multirow[t]{2}{*}{$\begin{array}{l}\text { Lancang Island, } \\
\text { Indonesia }\end{array}$} & $\begin{array}{l}\text { Periglypta reticulata } \\
\text { (muscle) }\end{array}$ & w.w. & 2018 & 0.26 & 0.04 & nd & 0.44 & 9.45 & 0.22 & 0.03 & 1.19 & 4.93 & This study \\
\hline & P. reticulata (digestive) & w.w. & 2018 & 0.46 & 0.19 & nd & 0.66 & 19.26 & 2.57 & 0.03 & 0.30 & 8.76 & This study \\
\hline Catania Gulf & $\begin{array}{l}\text { D. trunculus } \\
\text { (composite) }\end{array}$ & w.w. & 2012 & 0.33 & 0.07 & & & & & & & & [14] \\
\hline Cochin Area & $\begin{array}{l}\text { Villorita crypinoides } \\
\text { (composite) }\end{array}$ & w.w. & 2011 & 0.34 & 0.68 & & & & & & & 40 & {$[26]$} \\
\hline Shanghai & $\begin{array}{l}\text { Mactra chinensis } \\
\text { (composite) }\end{array}$ & d.w. & 2008 & & 0.13 & & & & & & & 14.8 & {$[16]$} \\
\hline Bohai Bay & $\begin{array}{l}\text { Mactra veneriformis } \\
\text { (soft tissue) }\end{array}$ & w.w. & 2008 & 1.00 & 0.37 & 0.04 & 1.26 & & & & & 11.57 & {$[17]$} \\
\hline Parit Jawa, Johor & $\begin{array}{l}\text { Polymesoda erosa } \\
\text { (total tissue) }\end{array}$ & d.w. & 2009 & 5.07 & 17.9 & & & & & & & 67.1 & {$[27]$} \\
\hline Parit Jawa, Johor & $\begin{array}{l}\text { Polymesoda erosa } \\
\text { (foot) }\end{array}$ & d.w. & 2009 & & 2.18 & & & & & & & 92.1 & {$[27]$} \\
\hline Parit Jawa, Johor & $\begin{array}{l}\text { Polymesoda erosa } \\
\text { (gill) }\end{array}$ & d.w. & & & 2.56 & & & & & & & 263 & {$[27]$} \\
\hline Telok Mas, Mallaca & $\begin{array}{l}\text { Polymesoda erosa } \\
\text { (total tissue) }\end{array}$ & d.w. & 2009 & 4.29 & 6.80 & & & & & & & 343 & {$[27]$} \\
\hline Telok Mas, Mallaca & $\begin{array}{l}\text { Polymesoda erosa } \\
\text { (foot) }\end{array}$ & d.w. & 2009 & & 0.937 & & & & & & & 105 & {$[27]$} \\
\hline Telok Mas, Mallaca & $\begin{array}{l}\text { Polymesoda erosa } \\
\text { (gill) }\end{array}$ & d.w. & 2009 & & 6.51 & & & & & & & 263 & [27] \\
\hline $\begin{array}{l}\text { Coast of New } \\
\text { Celedonia }\end{array}$ & $\begin{array}{l}\text { Gafrarium tumidum } \\
\text { (soft tissue) }\end{array}$ & w.w. & 2008 & & & & & & & & & & {$[28]$} \\
\hline $\begin{array}{l}\text { Coast of New } \\
\text { Celedonia }\end{array}$ & $\begin{array}{l}\text { Periglypta chemnitzi } \\
\text { (soft tissue) }\end{array}$ & w.w. & 2008 & & & & & & & & & & {$[28]$} \\
\hline Astamudi Lagoon & $\begin{array}{l}\text { Villorita cyprinoides } \\
\text { (soft tissue) }\end{array}$ & d.w. & 2017 & 2.42 & 1.46 & & & & & & & 76.79 & [29] \\
\hline $\begin{array}{l}\text { Catania Fish } \\
\text { Market }\end{array}$ & $\begin{array}{l}\text { D. trunculus } \\
\text { (composite) }\end{array}$ & w.w. & 2013 & 0.32 & 0.07 & & & & & & & 7.62 & [14] \\
\hline Marudu Bay & $\begin{array}{l}\text { Polymesoda expansa } \\
\text { (soft tissue) }\end{array}$ & d.w. & 2017 & 5.71 & 2.31 & & & & & & & 377.1 & {$[22]$} \\
\hline $\begin{array}{l}\text { Rias of Pontevedra } \\
\text { (A1) }\end{array}$ & $\begin{array}{l}\text { Dosiona exoleta (soft } \\
\text { tissue) }\end{array}$ & d.w. & 2006 & & 4.65 & & & & & & & 203 & {$[30]$} \\
\hline$(\mathrm{A} 2)$ & & d.w. & & & 2.45 & & & & & & & 176 & {$[30]$} \\
\hline (A4) & & d.w. & & & 7.89 & & & & & & & 301 & {$[30]$} \\
\hline $\begin{array}{l}\text { Kuala Kemaman, } \\
\text { Terengganu, }\end{array}$ & Polymesoda expansa & d.w. & 2016 & & 6.9 & & & & 15.9 & & & 12.8 & {$[34]$} \\
\hline Coastline of India & Polymesoda aerosa & d.w. & 2013 & 14 & 1.1 & & & & & & & 91.7 & {$[35]$} \\
\hline \multirow[t]{4}{*}{$\begin{array}{l}\text { Tanjung Lumpur, } \\
\text { Malaysia }\end{array}$} & $\begin{array}{l}\text { Polymesoda expansa } \\
\text { (soft tissue) }\end{array}$ & d.w. & 2017 & & 1.94 & & & & & & & 269 & {$[36]$} \\
\hline & NADFC (2018) & w.w. & 2018 & & 0.20 & & & & & & & & {$[31]$} \\
\hline & ANZFC & w.w. & 2000 & & 2 & & & & & & & & {$[32]$} \\
\hline & JECFA/WHO & w.w. & 2016 & & & & & & & & & & {$[33]$} \\
\hline
\end{tabular}


Sr, $\mathrm{Pb}(p<0.05)$ and As, $\mathrm{Co}, \mathrm{Ni}(p<0.01)$. Cd had only a significant correlation with Se in the muscle $(p<0.05)$, whereas $\mathrm{Pb}$ was negatively correlated with $\mathrm{V}$ in muscle but had a strong correlation between essential metals, $\mathrm{Fe}, \mathrm{Ni}$ $(p<0.05)$ and Co $(p<0.01)$ in digestive. Arsenic had strong correlations with $\mathrm{Co}, \mathrm{Ni}, \mathrm{Sr}(p<0.05)$, and $\mathrm{Cd}$ in digestive but only had a positive correlation with Co in muscle. Mercury $(\mathrm{Hg})$ also had a strong association with Co, Ni, Sr $(p<0.05)$ and with As and Cd $(p<0.01)$ in digestive but only strongly influenced As in muscle. Alonso et al. reported that there was a significant correlation between metal and essential metal, for example, $\mathrm{Cd}$ and was positively associated with most of the essential metals analyzed in the digestive, the organ that accumulates in higher concentrations, compared to muscle [25]. These interactions probably indicate that essential metals for homeostatic mechanisms in organisms, to regulate mineral balance in the body, could be interfered with or competed with toxic elements. This fact would bring a threat to the normal function of metal in the body. So, monitoring of toxic metals in the main compartment like sediment that transfers metals into marine organisms should be done regularly and intensively [26].

\section{- CONCLUSION}

This study investigated the concentrations of nineteen metals in muscle tissues and digestive contents of $P$. reticulata from Lancang Island, part of Seribu Islands. Concentrations of total Arsenic in muscle and digestive were found to exceed permissible limit from the National Agency of Drug and Food Control. Cadmium, Lead, and Mercury have shown under the permissible limit in muscle tissues and digestive contents, but $\mathrm{Cd}$ and $\mathrm{Pb}$ in the digestive contents should be a concern because it nearly reaches permissible limit from NADFC. Toxic metals $(\mathrm{Pb}, \mathrm{Hg}, \mathrm{Cd}$, and $\mathrm{As})$ showed strong correlations with several essential metals, so monitoring for particular metals must be done intensively. Because of the high accumulation in digestive content, the elimination of this part was suggested when consuming this clam to reduce bioaccumulation of heavy metals in the human body.

\section{- ACKNOWLEDGMENTS}

This research was financially funded by Demand Driven Research Grant (DDRG LIPI - COREMAP CTI) No: B-1191/IPK.2/KS.02/III/2018 the fiscal year 2018. The authors also acknowledge the financial support from Riset Prioritas COREMAP-CTI No.: B-5006/IPK. 2/KP.06/I/2019 year fiscal 2019 for the publication support.

\section{- AUTHOR CONTRIBUTION}

$\mathrm{S}$ as main contributor responsible for on data analysis, and drafted the manuscript. $\mathrm{RP}$ and $\mathrm{ZP}$ executed sampling and drafted the manuscript, while NS performed laboratory analysis.

\section{- REFERENCES}

[1] Tchounwou, P.B., Yedjou, C.G., Patlolla A.K., and Sutton, D.J., 2012, Heavy metal toxicity and environment, Exp. Suppl., 101, 133-164.

[2] WHO/FAO/IAEA, 1996, Trace elements in human nutrition and health, Word health organization, Geneva, Switzerland.

[3] Chang, L.W., Magos, L., and Suzuki, T., 1996, Toxicology of Metals, CRC Press, Boca Raton, FL, USA, 233-254.

[4] Inoue, K.I., 2013, Heavy metal toxicity, J. Clin. Toxicol., S3, 007.

[5] Ibrahim, N.K., and Abu El-Regal, M.A., 2014, Heavy metals accumulation in marine edible molluscs, Timsah Lake, Suez Canal, Egypt, ARPN J. Sci. Technol., 4 (4), 282-288.

[6] Hu, S., Su, Z., Jiang, J., Huang, W., Liang, X., Hu, J., Chen, M., Cai, W., Wang, J., and Zhang, X., 2016, Lead, cadmium pollution of seafood and human health risk assessment in coastline of the southern China, Stochastic Environ. Res. Risk Assess., 30 (5), 1379-1386.

[7] Langston, W.J., Bebianno, M.J., and Burt, G.R., 1998, "Metal handling strategic in mollusk" in Metal Metabolism in Aquatic Environments, Eds. Langston, W.J., and Bebianno, M.J., Chapman \& Hall, London, 219-283. 
[8] Abdallah, M.A.M., and Abdallah, A.M.A., 2008, Biomonitoring study of heavy metals in biota and sediments in South Eastern coast of Mediterranean sea, Egypt, Environ. Monit. Assess., 146, 139-145.

[9] Richard, G.P., 1988, Microbial purification of shellfish: A review of depuration and relaying, J. Food Prot., 51 (3), 218-251.

[10] Shirnesan, G., Bakhtiari, A.R., Seyfabadi, S.J., and Mortazavi, S., 2013, Environmental geochemistry of $\mathrm{Cu}, \mathrm{Zn}$ and $\mathrm{Pb}$ in sediment from Qeshm IslandPersian Gulf, Iran: A comparison between the northern and southern coast and ecological risk, Geochem. Int., 51 (8), 670-676.

[11] Dsikowitzky, L., Dwiyitno, Heruwati, E., Ariyani, F., Irianto, H.E., and Schwarzbauer, J., 2014, Exceptionally high concentrations of the insect repellent $N, N$-diethyl- $m$-toluamide (DEET) in surface waters from Jakarta, Indonesia, Environ. Chem. Lett., 12 (3), 407-411.

[12] SNI, 2006, Cara uji kimia-bagian 2: Penentuan kadar air pada produk perikanan, Badan Standardisasi Nasional-BSN, Jakarta, 1-12.

[13] USEPA, 2007, Method 3051A: Microwave assisted acid digestion of sediments, sludge, soils, and oils, https://www.epa.gov/sites/production/files/2015-12/ documents/3051a.pdf.

[14] Copat, C., Arena, G., Fiore, M., Ledda, C., Fallico, R., Sciacca, S., and Ferrante, M., 2013, Heavy metals concentrations in fish and shellfish from eastern Mediterranean Sea: Consumption advisories, Food Chem. Toxicol., 53, 33-37.

[15] Sivaperumal, P., Sankar, T.V., and Viswanathan Nair, P.G., 2007, Heavy metal concentrations in fish, shellfish and fish products from internal market of India vis-à-vis international standards, Food Chem., 102 (3), 612-620.

[16] Lei, B.L., Chen, L., Hao, Y., Cao, T.H., Zhang, X.Y., Yu, Y.G., and Fu, J.M., 2013, Trace elements in animal-based food from Shanghai markets and associated human daily intake and uptake estimation considering bio accessibility, Ecotoxicol. Environ. Saf., 96, 160-167.
[17] Li, Y., Liu, H., Zhou, H., Ma, W., Han, Q., Diao, X., and Xue, Q., 2015, Concentration distribution and potential health risk of heavy metals in Mactra veneriformis from Bohai Bay, China, Mar. Pollut. Bull., 97 (1-2), 528-534.

[18] Peto, M.V., 2010, Aluminium and iron in humans: Bioaccumulation, pathology and removal, Rejuvenation Res., 13 (5), 589-598.

[19] Dhanakumar, S., Solaraj, G., and Mohanraj, R., 2015, Heavy metals partitioning in sediments and bioaccumulation in commercial fish species of three major reservoirs of river cauvery delta region, India, Ecotoxicol. Environ. Saf., 113, 145-151.

[20] Moiseenko, T.I., and Kurdyasvtseva, L.S., 2001, Trace metal accumulation and fish pathologies in area affected by mining and metallurgical enterprises in the Kola Region, Russia, Environ. Pollut., 114 (2), 285-297.

[21] Waykar, B., and Deshmukh, G., 2012, Evaluation of bivalves as bio indicators of metal pollution in fresh water, Bull. Environ. Contam. Toxicol., 88, 48-53.

[22] Harsono, N.D.B.D., Ransangan, J., Denil, D.J., and Soon, T.K., 2017, Heavy metals in marsh clam (Polymesoda expansa) and green mussel (Perna viridis) along the northwest coast of Sabah, Malaysia, Borneo J. Mar. Sci. Aquacult., 01, 25-32.

[23] Liu, J., Cao, L., and Dou, S., 2017, Bioaccumulation of heavy metals and health risk assessment in three benthic bivalves along the coast of Laizhou Bay, China, Mar. Pollut. Bull., 117 (1-2), 98-110.

[24] Wei, H., Hou, L., Cui, Y., and Wei, Y., 2018, Effect of $\mathrm{Ti}$ content on corrosion behavior of $\mathrm{Cu}$-Ti alloys in $3.5 \% \mathrm{NaCl}$ solution, Trans. Nonferrous Met. Soc. China, 28 (4), 669-675.

[25] Alonso, M.L., Montaña, F.P., Miranda, M., Castillo, C., Hernández, J., and Benedito, J.L., 2004, Interactions between toxic ( $\mathrm{As}, \mathrm{Cd}, \mathrm{Hg}$ and $\mathrm{Pb}$ ) and nutritional essential ( $\mathrm{Ca}, \mathrm{Co}, \mathrm{Cr}, \mathrm{Cu}, \mathrm{Fe}, \mathrm{Mn}, \mathrm{Mo}$, $\mathrm{Ni}, \mathrm{Se}, \mathrm{Zn}$ ) elements in the tissues of cattle from NW Spain, BioMetals, 17, 389-397.

[26] Kalogeropoulos, N., Karavoltsos, S., Sakellari, A., Avramidou, S., Dassenakis, M., and Scoullos, M., 
2012, Heavy metals in raw, fried and grilled Mediterranean finfish and shellfish, Food Chem. Toxicol., 50 (10), 3702-3708.

[27] Edward, F.B., Yap, C.K., Ismail, A., and Tan, S.G., 2009, Interspecific variation of heavy metal concentrations in the different parts of tropical intertidal bivalves, Water Air Soil Pollut., 196, 297-309.

[28] Chouvelon, T., Warnau, M., Churlaud, C., and Bustamante, P., 2009, Hg concentrations and related risk assessment in coral reef crustaceans, molluscs and fish from New Caledonia, Environ. Pollut., 157 (1), 331-340.

[29] Ragi, A.S., Leena, P.P., Cheriyan, E., and Nair, S.M., 2017, Heavy metal concentrations in some gastropods and bivalves collected from the fishing zone of South India, Mar. Pollut. Bull., 118 (1-2), 452-458.

[30] Sánchez-Marín, P., and Beiras, R., 2008, Lead concentrations and size dependence of lead accumulation in the clam Dosinia exoleta from shellfish extraction areas in the Galician Rias (NW Spain), Aquat. Living Resour., 21 (1), 57-61.

[31] NADFC, 2018, Batas maksimum cemaran logam berat dalam pangan olahan, Peraturan Badan Pengawas Obat dan Makanan No. 5, Jakarta, 1-15.

[32] ANZFC, 2012, Standard 1.4.1. Contaminants and natural toxicants, Australia New Zealand Food Standards Code, 1-7, https://www.legislation.gov. au/Details/F2011C00542/Download.

[33] CODEX ALIMENTARIUS, 2016, General standard for contaminants and toxin in food and feed, CODEX STAND 193-1995, 1-65.

[34] Dabwan, A.H.A., and Taufik, M., 2016, Bivalves as bio-indicators for heavy metals detection in Kuala Kemaman, Terengganu, Malaysia, Indian J. Sci. Technol., 9 (9), 88708.

[35] Gawade, L., Chari, N.V.H., Sarma, V.V., and Ingole, B.S., 2013, Variation in heavy metals concentration in the edible oyster Crassostrea madrasensis, clam Polymesoda erosa and grey mullet Liza aurata from coastline of India, Indian J. Sci., 2 (4), 59-63.

[36] Chuan, O.M., and Ibrahim, A., 2017, Determination of selected metallic elements in marsh clam, Polymesoda expansa, collected from Tanjung Lumpur Mangrove Forest, Kuantan, Pahang, Borneo J. Mar. Sci. Aquacult., 01, 65-70. 